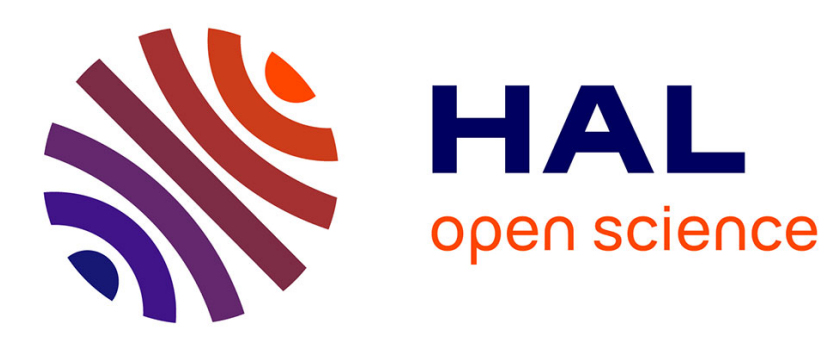

\title{
Mean-field theory of nuclear stability and exotic point-group symmetries
}

J Dudek, A Góźdź, K Mazurek, H Molique

\section{To cite this version:}

J Dudek, A Góźdź, K Mazurek, H Molique. Mean-field theory of nuclear stability and exotic pointgroup symmetries. Journal of Physics G: Nuclear and Particle Physics, 2010, 37 (6), pp.064032. 10.1088/0954-3899/37/6/064032 . hal-00600811

\section{HAL Id: hal-00600811 \\ https://hal.science/hal-00600811}

Submitted on 16 Jun 2011

HAL is a multi-disciplinary open access archive for the deposit and dissemination of scientific research documents, whether they are published or not. The documents may come from teaching and research institutions in France or abroad, or from public or private research centers.
L'archive ouverte pluridisciplinaire HAL, est destinée au dépôt et à la diffusion de documents scientifiques de niveau recherche, publiés ou non, émanant des établissements d'enseignement et de recherche français ou étrangers, des laboratoires publics ou privés. 


\title{
Mean-field theory of nuclear stability and exotic point-group symmetries
}

\author{
J DUDEK ${ }^{1}$, A GÓŹDŹ ${ }^{2}$, K MAZUREK $^{3}$ and \\ H MOLIQUE ${ }^{1}$ \\ ${ }^{1}$ Institut Pluridisciplinaire Hubert Curien \\ Département de Recherches Subatomiques UMR7178, IN2P3-CNRS and \\ Université de Strasbourg, 23 rue du Loess B.P. 28, \\ F-67037 Strasbourg Cedex 2, France \\ 2Zakład Fizyki Matematycznej, Uniwersytet Marii Curie-Skłodowskiej, \\ pl. Marii Curie-Skłodowskiej 1, PL-20031 Lublin, Poland \\ ${ }^{3}$ The Niewodniczański Institut of Nuclear Physics, Polish Academy of Sciences, \\ ul. Radzikowskiego 152, Pl-31-342 Kraków, Poland \\ and Grand Accélérateur National d'Ions Lourds, Bd Henri Becquerel \\ BP 55027 - 14076 Caen Cedex 05, France \\ E-mail: Jerzy.Dudek@IReS.in2p3.fr, Andrzej.Gozdz@umcs.lublin.pl, \\ Katarzyna.Mazurek@ifj.edu.pl, Herve.Molique@IReS.in2p3.fr
}

\begin{abstract}
.
We formulate the principles of the mean-field theory of nuclear stability employing the point-group, and group-representation theories. The related pointgroup hierarchy of importance in the context of nuclear stability is constructed and discussed. We introduce the notion of the magic-number chains associated with each symmetry - in analogy to the spherical-symmetry nuclear magic-numbers. To prepare the criteria for the experimental search of introduced symmetries we examine the simplified collective rotation-vibration model whose Hamiltonian is invariant under the symmetries in question. We illustrate the construction of the solutions that form at the same time irreducible-representations of the pointgroups in question - in view of formulating the experimental symmetry-criteria through the application of the branching-ratio techniques. Since the criteria may involve very weak transitions whose experimental research may be at the limit of the present-day experiments, the desires may arise, as it was the case in the past, to replace the difficult experiments by an inadequate modelling. In this context we present an alert: The use of oversimplified quantum mechanics exercises in place of experiments and/or microscopic theories is likely to produce meaningless results.
\end{abstract}

PACS numbers: 21.60.-n,21.10.-k,21.10.Pc 


\section{Introduction: Nuclear stability and point-group symmetries}

Studying exotic nuclear systems with extreme numbers of neutrons for any fixed proton number represents one of the most important research directions at the large scale nuclear physics facilities world-wide. One of the central issues in this research is the question of stability (thus of life-times) of the investigated nuclear species, the shorter the life-times the bigger the problems with the production and, importantly, detection at a conclusive level of statistics. It therefore becomes clear that realistic modelling of nuclear stability on the theory grounds will play a very important role, in particular, in determining both the strategies as well as the detailed planing of experiments.

The stability of a nuclear system is related to the nucleonic binding, thus to the presence of large gaps in the single-nucleonic spectra: to an approximation, the larger the energy gaps at the Fermi level the stronger the stability. Contemporary effective Hamiltonians such as nuclear mean-field Hamiltonians can generate information of this type in a realistic manner. Since the majority of nuclei are non-spherical, the well established capacity of the mean-field theories to describe the nuclear spontaneous spherical-symmetry breaking, and by the same token, the nuclear deformations, is of considerable advantage. Nuclear shapes are directly related to the spatial properties of the underlying mean-field Hamiltonians, whereas the latter determine the nucleonic energy spectra. This link opens up the possibilities of analyzing the global properties of nucleonic spectra, such as energy-level degeneracies, fluctuations of the level densities etc. through the symmetries of the mean-field Hamiltonians directly. Indeed, through employing powerful group- and group-representation theories that help to predict the most likely presence of gaps in the single particle spectra prior to the detailed calculations we may obtain, as it will be shown, instructive guide-lines for such large scale calculations, cf. section 2, especially table 1 and figure 1.

In this article we formulate the principal lines of the symmetry-based research of stability of exotic nuclear shapes. The term 'exotic' is arbitrarily used to denote any symmetry other than spherical, prolate-, oblate- or triaxial-quadrupole as well as axial-octupole geometries. We present general group-theoretical arguments adapted to the form of the mean-field theory Hamiltonians as well as the collective rotationvibration Hamiltonians. It will be shown that the latter present certain mathematical advantages when formulating the criteria for the experimental identification of the symmetries, but the corresponding signals may be weak and detection difficult.

In this article we discuss a number of Open Problems related to nuclear pointgroup (geometrical) symmetries whose hierarchy is presented in section 2 . We have verified on the examples of tetrahedral and octahedral symmetries which, for reasons explained below are on top of the list, that using realistic Hartree-Fock Bogoliubov and phenomenological methods one obtains the high-symmetry solutions for nuclei in numerous areas of the Periodic Table. We believe this is not so much the question of 'Whether?' but 'Where in the Nuclear Chart exotic symmetries can be found?' A number of Open Problems relates to 'What to look for?' In section 3 we present group-theory considerations that may help in the dedicated experimental studies.

Finally, we formulate a few brief comments on the in-adapted approaches to look for the symmetries in nuclear systems which, very unfortunately, are applied by certain authors leading to totally wrong conclusions based on possibly technically correct an experimental input. Interested reader is referred to the article focussing on the complete description of the problem and the underlying techniques in [1]. 


\section{Symmetry-based mean-field theory of nuclear stability}

Let us begin by a few facts from the group-representation theory relating the symmetry of the Hamiltonian, $H$, and the degeneracy of levels. Let group $G=\left\{\hat{g}_{1}, \hat{g}_{2}, \ldots \hat{g}_{f}\right\}$ be a symmetry group of $H$, what implies that a representation $D(\hat{g})$ exists such that $[D(\hat{g}), H]=0, \forall \hat{g} \in G$. Suppose that $D_{1}, D_{2}, \ldots D_{r}$ are the irreducible representations of $G$ with dimensions $d_{1}, d_{2}, \ldots d_{r}$, respectively. Then the spectrum of $H$ splits into multiplets with degeneracies either equal $d_{1}$, or $d_{2}$ or $\ldots d_{r}$. The spherical symmetry provides a particular example: the irreducible representations are characterised by the single-particle angular momentum quantum numbers $j$, the implied Hamiltonian block-sub-matrices have the dimensions $(2 j+1) \times(2 j+1)$ and the degeneracies are equal to $d_{j}=(2 j+1)$. We will argue that the irreducible representations of the symmetry groups influence spectral properties through two characteristics: both the dimensions $\left\{d_{k}: k=1, \ldots r\right\}$ and the total number of irreducible representations, $r$.

Table 1. Double point groups together with the numbers of their irreducible representations and dimensions. The information in the last column should be read as follows. For instance, in the case of the double octahedral point group $O_{h}^{D}$ we find 4 two-dimensional and 2 four-dimensional irreducible representations.

\begin{tabular}{||c|c|c|c||}
\hline No. & Group & No. Irr. & Dimensions \\
\hline 1 & $O_{h}^{D}$ & 6 & $4 \times 2 \mathrm{D}$ and $2 \times 4 \mathrm{D}$ \\
2 & $T_{d}^{D}$ & 3 & $2 \times 2 \mathrm{D}$ and $1 \times 4 \mathrm{D}$ \\
\hline 3 & $C_{6 h}^{D}$ & 12 & $12 \times 1 \mathrm{D}(6 \times 2 \mathrm{D})$ \\
4 & $D_{6 h}^{D}$ & 6 & $6 \times 2 \mathrm{D}$ \\
\hline 5 & $C_{4 h}^{D}$ & 8 & $8 \times 1 \mathrm{D}(4 \times 2 \mathrm{D})$ \\
6 & $D_{4 h}^{D}$ & 4 & $4 \times 2 \mathrm{D}$ \\
\hline 7 & $D_{3 h}^{D}$ & 3 & $3 \times 2 \mathrm{D}$ \\
8 & $C_{6 v}^{D}$ & 3 & $3 \times 2 \mathrm{D}$ \\
9 & $D_{6}^{D}$ & 3 & $6 \times 2 \mathrm{D}$ \\
\hline 10 & $C_{6}^{D}$ & 6 & $6 \times 1 \mathrm{D}(3 \times 2 \mathrm{D})$ \\
11 & $S_{6}^{D}$ & 6 & $6 \times 1 \mathrm{D}(3 \times 2 \mathrm{D})$ \\
12 & $C_{3 h}^{D}$ & 6 & $6 \times 1 \mathrm{D}(3 \times 2 \mathrm{D})$ \\
13 & $C_{3 i}^{D}$ & 6 & $2 \times 2 \mathrm{D}($ reference $)$ \\
\hline 14 & $D_{2 h}^{D}$ & 2 &
\end{tabular}

Point groups that express geometrical symmetries in quantum mechanics are classified as 'simple' or 'double', the latter applicable to the Fermion spaces and thus to the mean-field Hamiltonians for nucleons. The names of the double groups are marked with the superscript $D$. The point-groups of interest in this article are listed in table 1 according to decreasing dimensions of their irreducible representations - as well as the decreasing numbers of irreducible representations. These two characteristics of various groups are compared with the ones of $D_{2 h}^{D}$, the symmetry of the so-called 'triaxial' 
nuclei, usually described with the help of quadrupole elongation $(\beta)$ and triaxiality $(\gamma)$ deformations. It is suggested in sections 2.1 and 2.2 that the bigger the numbers of irreducible representations and larger their dimensions per given symmetry group, the larger the chances for strong inter-spacing of levels.

\subsection{Symmetry groups and the effect of a large number of irreducible representations}

To our knowledge, there exist no rigorous mathematical argument connecting e.g. the gaps or the maximum-gaps in the energy spectra with the characteristics of the irreducible representations of symmetry groups. We have performed numerous tests using the realistic mean-field models to convince ourselves that the following properties are approximately valid (so far $O_{h}^{D}, T_{d}^{D}$ and $C_{4 h}^{D}$ groups were tested), cf. figure 1.

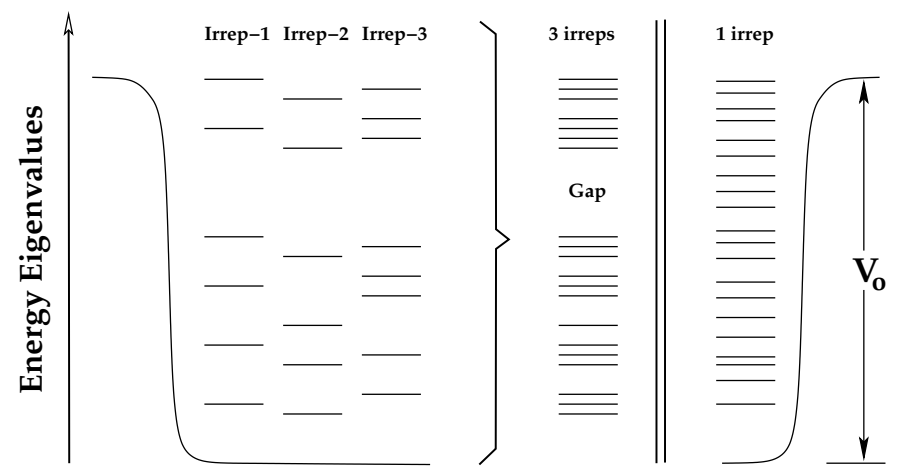

Figure 1. Schematic: 20 levels in a potential well; for comments cf. the text.

To introduce a reference mechanism let us consider a symmetry group possessing only one two-dimensional or two-dimensional equivalent $\ddagger$ irreducible representation leading to Kramers degeneracy. Hamiltonians with the implied symmetry generate two matrix-blocks, $[H]$ and $\left[H^{*}\right]$, and it follows from the hermiticity that the corresponding spectra are identical. With increasing deformation which preserves that symmetry (the nuclear shapes resembling less and less a sphere) the levels have a tendency to fill in the energy window $V_{o}$ tending towards a uniform distribution (figure 1, right).

For symmetry groups with $r$ irreducible representations the mechanism of filling in the energy window $V_{o}$ repeats itself within each of the $r$ blocks. Level-density fluctuations leading to relatively irregular spacings are stronger since there are much fewer levels per each block in this case (figure 1 left). The mechanism presented here qualitatively leads to strong non-spherical symmetry "magic" gaps in the single particle spectra with the accompanying strong shell effects that lead to prediction of possible shape-coexisting minima in various areas of the Periodic Table, cf. [6].

\subsection{Symmetry groups and the effect of dimensions of irreducible representations}

Dimensions of the irreducible representations are equal to the degeneracies of related levels, as e.g. $(2 j+1)$-degeneracy in spherical nuclei. Suppose there are $N_{b}$ bound

$\ddagger$ The mathematical details about the Wigner theorem classifying the irreducible representations as potentially real, pseudo-real and essentially complex, the mathematical terminology that we will not need here, can nevertheless be found in [2]. 
states in a deformed potential of depth $V_{o}$. Formally, we can place $2 \cdot N_{b}$ particles in the potential and the average level spacing $\langle d\rangle \sim V_{o} /\left(N_{b}\right)$. The $2 \cdot N_{b}$ particles can be put much more 'economically' if several levels carry degeneracies higher than 2 - this is because the same number of particles can be placed on the number of levels $N_{d e g}$ which is smaller than $N_{b}$, so that the average level spacing $\left\langle d_{d e g}\right\rangle \sim V_{o} /\left(N_{d e g}\right)$ will be larger (or considerably larger) than the one in the case of no extra degeneracies.

There are only a few double point groups that have dimensions of their irreducible representations larger than 2. These are first of all the octahedral and tetrahedral groups listed as the first two in table 1 . The icosahedral group which belongs to the same category generates regular shapes probably too close to the spherical form to be of interest in the nuclear physics applications; so far, arbitrarily, we have disregarded this group. However, the degeneracy arguments (equivalently: dimensions of irreducible representations) together with the arguments of the large number of irreducible representations place the octahedral and tetrahedral groups on top of the list of interest in the context of strong non-spherical shell-gaps and an implied increase in nuclear stability at the corresponding particle numbers, see below.

An illustration of this qualitative discussion in the context of the realistic nuclear calculations will be given in section 2.4 after having introduced the solution of the non-trivial problem of constructing the mean-field Hamiltonians preserving an a priori given (pre-defined) point-group symmetry.

\subsection{Construction of the symmetry-preserving mean-field Hamiltonians}

Focusing on the mean-field applications, suppose that the considered Hamiltonian depends on an ensemble of parameters $\{\alpha\}$ specifying nuclear shapes, thus $e_{n}=e_{n}(\alpha)$.

2.3.1. Phenomenological Mean Field Hamiltonians: Woods-Saxon or Yukawa folded. Consider first a deformed Woods-Saxon or Yukawa-folded mean-field potential, with the nuclear surface $\Sigma$ represented with the help of spherical harmonics $Y_{\lambda \mu}$ as

$$
\Sigma: \quad R(\vartheta, \varphi)=c(\{\alpha\})\left[1+\sum_{\mu=-\lambda}^{\lambda} \alpha_{\lambda \mu}^{\star} Y_{\lambda \mu}(\vartheta, \varphi)\right]
$$

where function $c(\alpha)$ assures the constant volume condition. Given symmetry group $G=\left\{\hat{g}_{1}, \hat{g}_{2}, \ldots \hat{g}_{f}\right\}$; by acting with any of the symmetry operations $\hat{g}_{i}$ on the nuclear surface we must obtain an image coinciding with the original i.e.

$$
\sum_{\lambda=2}^{\lambda_{\max }} \sum_{\mu=-\lambda}^{\lambda} \alpha_{\lambda \mu}^{\star}\left[\hat{g}_{i} Y_{\lambda \mu}(\vartheta, \varphi)\right]=\sum_{\lambda=2}^{\lambda_{\max }} \sum_{\mu=-\lambda}^{\lambda} \alpha_{\lambda \mu}^{\star} Y_{\lambda \mu}(\vartheta, \varphi), \quad \forall \hat{g}_{i} .
$$

Let us introduce the Euler-angle representation of group elements adapted to act on the spherical harmonics: $\hat{g}_{i} \rightarrow \Omega_{i}$ where $\Omega_{i} \equiv\left\{\omega_{1}, \omega_{2}, \omega_{3}\right\}$ are the triplets of Euler angles, cf. [2]; e.g. for a four-fold $\mathcal{O}_{z}$-axis, $\Omega=\{\pi / 2,0,0\}$. One can show, [3], that deformation parameters $\alpha_{\lambda \mu}$ obeying the symmetry $G$ satisfy in each order $\lambda$ :

$$
\sum_{\mu=-\lambda}^{\lambda}\left[(-1)^{\eta_{i}}(-1)^{\lambda} D_{\mu^{\prime} \mu}^{\lambda}\left(\Omega_{i}\right)-\delta_{\mu \mu^{\prime}}\right] \alpha_{\lambda \mu}^{\star}=0 \text { for } i=1,2, \ldots f,
$$

where $\eta_{i}=0$ (or 1 ) depending on whether the inversion is not (or is) involved. This system of $f$ equations resembles the system of eigenvalue problems with eigenvalues equal to unity. Since equations in (3) are uniform, multiplying their solution $\bar{\alpha}_{\lambda \mu}$ by 
a constant generates another solution. This property allows to select, e.g. $\bar{\alpha}_{\lambda \mu=0}$ as an independent parameter and define a 'hyper-curve' $\bar{\alpha}_{\lambda \mu}=\bar{\alpha}_{\lambda \mu}\left(\bar{\alpha}_{\lambda \mu=0}\right)$; for higher multipolarites bi- or multi-dimensional hyper-surfaces are obtained provided the solutions of the system in (3) exits at all. In fact, the system in question is over-defined - it contains more equations than unknowns. The fact that solutions (sometimes) exist is a result of the symmetry. Since all the elements of a group can be constructed out of generators, in practice we need to solve only the systems of equations for the generators.

Examples of solutions for the tetrahedral and octahedral symmetries are given by equations (4)-(9) below. For instance, for the tetrahedral symmetry the solutions exist only for odd $\lambda \geq 3$, with the exception of $\lambda=5$; we have [4]:

$$
\begin{aligned}
& \lambda=3: \quad \alpha_{3, \pm 2} \equiv t_{3} ; \quad \lambda=5: \text { no solution exists; } \\
& \lambda=7: \quad \alpha_{7, \pm 2} \equiv t_{7} \text { and } \alpha_{7, \pm 6} \equiv-\sqrt{\frac{11}{13}} t_{7} ; \\
& \lambda=9: \quad \alpha_{9, \pm 2} \equiv t_{9} \text { and } \alpha_{9, \pm 6} \equiv+\sqrt{\frac{28}{198}} t_{9}, \quad \text { etc. }
\end{aligned}
$$

Observe the presence of the characteristic geometrical factors which are necessary to guarantee that the symmetry of the nuclear surfaces is obeyed at each value of $t_{7}, t_{9}$, etc. The solutions for the octahedral symmetry exist for even $\lambda \geq 4$ and we find

$$
\begin{aligned}
& \lambda=4: \quad \alpha_{4,0} \equiv o_{4} \quad \text { and } \quad \alpha_{4, \pm 4} \equiv-\sqrt{\frac{5}{14}} o_{4} \\
& \lambda=6: \quad \alpha_{6,0} \equiv o_{6} \quad \text { and } \quad \alpha_{6, \pm 4} \equiv+\sqrt{\frac{7}{2}} o_{6},
\end{aligned}
$$

together with

$$
\begin{aligned}
\lambda=8: \quad \alpha_{8,0} \equiv o_{8} \quad \text { and } \quad \alpha_{8, \pm 4} \equiv+\sqrt{\frac{28}{198}} o_{8}, \\
\text { and } \quad \alpha_{8, \pm 8} \equiv+\sqrt{\frac{65}{198}} o_{8}, \text { etc. }
\end{aligned}
$$

Let us notice a property of a certain mathematical elegance: for the multipolarity orders $\lambda \leq \lambda_{\max }=9$ studied we have the symmetries generated by one-parameter families of (hyper-)curves in the deformation space and moreover, the multipolarities $\lambda$ are never mixed what simplifies the technical applications of the formalism.

To our knowledge tetrahedral and octahedral symmetries are the only ones for which the problem of generating mean-field Hamiltonians invariant with respect to a predefined symmetry has been examined and solved; the solutions for most of the other point groups in table 1 belong to the domain of Open Problems, although the simple formalism summarized by equations (1)-(9) paves the way towards this goal.

2.3.2. Microscopic Mean-Fields - Hartree-Fock and Density-Functional Hamiltonians. All what has been said about the symmetry-respecting combinations of the $\bar{\alpha}_{\lambda \mu}$ coefficients in each given order $\lambda$ for the Woods-Saxon and Yukawa-folded potentials can be translated into the Hartree-Fock and constrained Hartree-Fock formalisms after replacing $\bar{\alpha}_{\lambda \mu}$ by multipole-moment constraints $\bar{Q}_{\lambda \mu}$. For instance, for the octahedral symmetry of the rank $\lambda=4$ we would search for solutions for which $\bar{Q}_{4, \pm 4} \equiv-\sqrt{5 / 14} \bar{Q}_{4}$, all other moments being either equal to zero or obeying the relations of the type in (8)-(9) for higher permitted $\lambda$-ranks.

The geometrical formalism introduced in the preceding section is extremely powerful in studying the symmetries of the self-consistent solutions as it can be seen 
Table 2. The Skyrme SIII Hartree-Fock-Boglyubov calculation results for tetrahedral solutions in light Rare-Earth nuclei using the zero-range densitydependent mixed pairing force in the pairing channel (based on [3]). Columns 3,4 and 5 give the multipole moments indicated. Column 6 shows the symmetry test based on relation (7); observe that the high precision equality between the results in columns 5 and 6 is a measure of an (almost) perfect symmetry.

\begin{tabular}{cccccc}
$\mathrm{Z}$ & $\mathrm{N}$ & $\begin{array}{c}Q_{32} \\
\left(b^{3 / 2}\right)\end{array}$ & $\begin{array}{c}Q_{40} \\
\left(b^{2}\right)\end{array}$ & $\begin{array}{c}Q_{44} \\
\left(b^{2}\right)\end{array}$ & $\begin{array}{c}-Q_{40} \times \sqrt{\frac{5}{14}} \\
\left(b^{2}\right)\end{array}$ \\
\hline & & & & & \\
64 & 86 & 0.94181 & -0.22737 & 0.13587 & 0.13588 \\
64 & 90 & 1.39465 & -0.42825 & 0.25592 & 0.25592 \\
64 & 92 & 0.00000 & -0.44721 & 0.26726 & 0.26726 \\
62 & 86 & 0.48739 & -0.08694 & 0.05195 & 0.05195 \\
62 & 88 & 0.81210 & -0.21880 & 0.13076 & 0.13076 \\
62 & 90 & 1.20601 & -0.38033 & 0.22729 & 0.22729
\end{tabular}

from the results in table 2 for the Hartree-Fock-Boglyubov calculations for a number of nuclei in the Rare Earth region. The results correspond to the unconstrained minimisation: the starting configuration has been constructed in such a way that no predefined symmetry was present in the wave-functions at the zero Hartree-Fock iteration, although a component of the tetrahedral symmetry has been present in the "mixed-input". As a consequence the convergence to the minima, as a matter of fact significantly below the energy at the spherical shapes of the considered nuclei, was assured just by the unconstrained variational principle. Observe also that solution for ${ }^{156} \mathrm{Gd}$ has a purely octahedral character, the symmetry $O_{h}^{D}$, whereas in all other solutions we have a combination of tetrahedral (weaker) and octahedral (stronger) symmetries, $T_{d}^{D} \subset O_{h}^{D}$, leading to the final $T_{d}^{D}$ (i.e. weaker) symmetry for all but ${ }^{156} \mathrm{Gd}$ nuclei. (For more details about the group-subgroup relations, cf. section 2.3.3.)

Let us emphasize that the unprepared user of the Hartree-Fock formalism would not recognize the presence of the higher-level symmetries without relations (4)-(9), as illustrated through comparison of columns 5 and 6 in table 2 for the tetrahedral and octahedral symmetries. It is a great advantage of the simple approach presented in section 2.3.1 that the results obtained with the e.g. Hartree Fock (and for that sake any other density-functional) formalism can be easily recognized and studied by using the multipole moments of the self-consistent solutions. The underlying geometrical formalism is in fact a common factor between the phenomenological and the densityfunctional methods. Preliminary studies in this direction have been advanced for the octahedral and tetrahedral groups and the interested reader is referred to the papers quoted above. However no systematic calculations for these two groups and no calculations for all other groups listed in the table 1 exist today and the implied studies belong to the Open Problem series.

2.3.3. Group vs. subgroup relations and symmetry-preserving energy minimisation. It will be instructive to present the role of the weaker i.e. less symmetric (more precisely: a subgroup) in the pair $T_{d}^{D} \subset O_{h}^{D}$ groups. The corresponding geometrical shapes are those of a triangle-base 'pyramid' and a 'diamond' shapes, respectively. And yet, the tetrahedral group is a sub-group of the octahedral one: in other words, 
the shapes which are octahedral-symmetric are also tetrahedral symmetric and the combination of the two classes of shapes may (and according to our tests does) enhance strong shell effects of the over-all tetrahedral symmetry, thus increasing the chances of the presence of the weaker among the two symmetries in atomic nuclei.

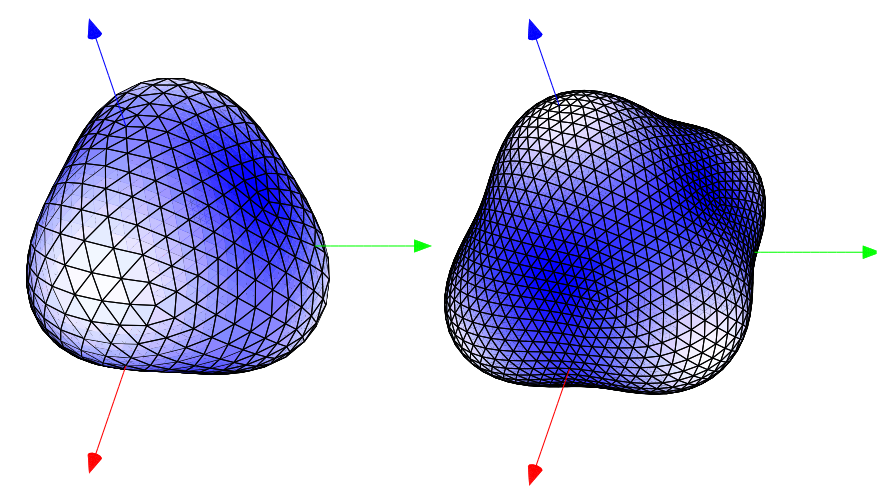

Figure 2. A nuclear 'pyramid' (left) and a nuclear 'diamond' (right) shapes corresponding to the tetrahedral and octahedral deformations with $t_{3}=0.2$ and $o_{4}=0.2$, respectively, cf. (4) and (7). It is usually recognized as not intuitive that so qualitatively different shapes belong to the common, 'weaker', tetrahedral symmetry and when combined may decrease considerably a energy of the tetrahedral-symmetric nucleus.

We find it instructive to compare the geometrical differences between shapes generated by the two discussed symmetry groups and yet, when combined together, leading to the symmetry of the subgroup, cf. figure 2. This simple observation is of a very high importance when minimising the energies with e.g. the very performant phenomenological models - and yet systematic studies of this type are only at the beginning, thus enlarging the list of the related Open Problems..

\subsection{Exotic symmetry groups and generalized magic gap chains}

Let us emphasize at this point that the term magic numbers usually employed in relation to the large gaps at spherical shapes corresponding to proton and/or neutron numbers $8,20,28,50,82$ and 126 should by no means be reserved to the $\mathrm{O}(3)$ group. To the contrary, we suggest that any point-group symmetry may generate its own series of magic numbers (we call them generalized magic gap chains) - with an important difference compared to the spherical symmetry case. Whereas a given nucleus cannot be more spherical than the other, it may be more (or less) deformed with the deformation belonging to e.g. tetrahedral-symmetry shapes. In other words, we may introduce the notion of tetrahedral-symmetry magic gaps by examining the single-particle spectra in function of multidimensional paths parametrised with the help of variables $t_{3}-t_{9}$ of (4-6) and/or $o_{\lambda}$ of various orders. The maximal gaps in the single-particle spectra do depend, individually, on deformation parameters. The gaps at the particle numbers $N_{i}$ correspond to the sets of symmetry-preserving deformation parameters $\mathcal{P}_{i} \equiv\left\{\bar{t}_{3}, \bar{t}_{7}, \bar{t}_{9}, \ldots\right\}_{i}$ - the generalized magic gap chains for the octahedral group are then characterized by the sets of numbers $\left\{N_{i}, \mathcal{O}_{i}\right\}$.

More generally, consider an arbitrary symmetry-group $G$ with the symmetryconserving parameters $\bar{\alpha}_{\lambda}^{G}$. By examining the single particle spectra in the spaces 
of $\left\{\bar{\alpha}_{\lambda}^{G}\right\}$ we obtain the magic gaps corresponding to the occupation numbers $N_{i}^{G}$ at deformations $\mathcal{P}_{i}^{G} \equiv\left\{\bar{\alpha}_{\lambda}^{G}\right\}_{i}$, so that finally the generalized magic gap chains are given by the ensembles of numbers $\left\{N_{i}^{G}, \mathcal{P}_{i}^{G}\right\}$. Properties of this type have been established for the tetrahedral symmetry group in [3]-[8] and references therein, but the systematic analysis for the other symmetry groups remains an Open Problem.

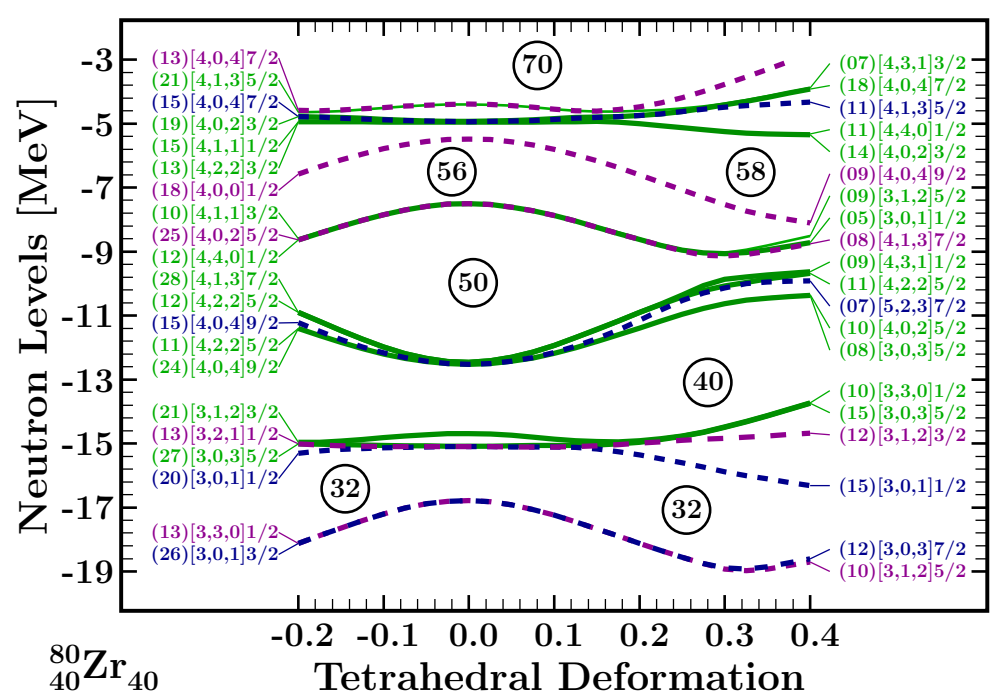

Figure 3. An example of realistic calculations with the phenomenological WoodsSaxon potential (here: Zirconium region) illustrating the mechanism of creating strong "tetrahedral" gaps in the single-particle spectra that can be comparable in size with the "spherical" gaps. The name "tetrahedral deformation" applies to $t_{3}$ of (4). The full lines represent solutions belonging to the four-dimensional irreducible representations while the dashed lines - the two remaining inequivalent two-dimensional representations. We use the Nilsson labelling of the curves, the numbers in parentheses being percentages of the dominating (Nilsson) label.

Figure 3 illustrates the powerful impact of the symmetry-considerations on generating large gaps in the realistic single-nucleon spectra. As it can be seen from the figure the tetrahedral-symmetry "magic" gap at $Z=40$ is comparable with the $Z=50$ spherical gap. Using this type of calculations the chain of the tetrahedral magic numbers has been found with $\mathrm{Z}, \mathrm{N}=32,40,56,70,90$ and $\mathrm{N}=136$. Similar diagrams in which the single-nucleon energies are drawn in a way allowing to distinguish between irreducible representations for various symmetry groups, cf. table 1, do not exist in the nuclear physics literature and represent a series of Open Problems for the future.

There exists yet another difference between the nuclear stability associated with spherical magic-gaps as compared to the generalized magic gaps. Whereas spherical configurations offer the most optimal surface- to Coulomb-energy ratio the nonspherical magic gaps may correspond to the forms whose surface- to Coulomb ratio of energies becomes extremely unfavourable - in other words: the corresponding total nuclear energies may lie very high, or, for the deformations large enough there may be no stable minima in the total energy landscapes corresponding to the magic gaps representing only the mean-field (and not the total nuclear energy) properties. These and related stability questions belong to the realm of Open Problems today. 


\section{Properties of rotational bands and criteria of detection of symmetries}

We will limit our considerations to the coexisting nuclear states with tetrahedral and quadrupole shapes as examples of 'exotic'- and 'usual' non-spherical symmetries, for which nuclear orientations in space and thus rotational bands can be defined. It has been shown using the methods of the preceding Section that the tetrahedral symmetry can be parametrised, to the lowest order, by $\alpha_{32}$ multipole deformation under the condition that all other (static) deformations are zero. Moreover, it can easily be shown that at the static tetrahedral minima $Q_{2, \mu}=0$, whereas all other multipole components $\alpha_{3, \mu \neq 2}$ lead to non-zero quadrupole moments. Within a simplified interpretation this implies that the E2 transitions within the bands of exact tetrahedral symmetry should vanish whereas in the case of a partial symmetry breaking they could be considerably smaller than the ones in the usual quadrupole-deformed nuclei $\S$. This implies that the usually neglected mechanisms such as e.g. the zero-point motion around the vanishing equilibrium deformations must not be neglected $\|$ anymore, e.g. the zero-point motion in other octupole degrees of freedom, $\alpha_{3, \mu \neq 2}$, as well as the quadrupole ones thus leading to, possibly less hindered, E2 transitions in the tetrahedral bands, cf. (28).

Let us introduce an intrinsic reference frame $\Sigma_{r o t}$ and consider the Hamiltonian:

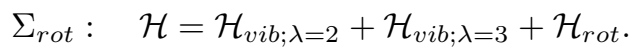

Since the spherical tensors $\alpha_{\lambda \mu}$ are in general complex, the usual way to proceed is to express the vibrational Hamiltonians using real parameters $a_{\lambda \mu}$ and $b_{\lambda \mu}$ defined by $\alpha_{\lambda \mu} \equiv a_{\lambda \mu}+i b_{\lambda \mu}$. Above we assume the simplest decoupled-vibration picture, where vibration potentials have the form $\frac{1}{2} C_{\lambda \mu}^{a}\left(a_{\lambda \mu}-a_{\lambda \mu}^{e q \cdot}\right)^{2}$ and/or $\frac{1}{2} C_{\lambda \mu}^{b}\left(b_{\lambda \mu}-b_{\lambda \mu}^{e q \cdot}\right)^{2}$ and the single-variable vibration Hamiltonians have the structure

$$
\mathcal{H}_{v i b}=-\frac{\hbar^{2}}{2 B} \frac{\partial^{2}}{\partial \rho^{2}}+\frac{1}{2} C\left(\rho-\rho^{e q}\right)^{2} \text { with } \rho \leftrightarrow a_{\lambda \mu} \text { or } b_{\lambda \mu}
$$

where $B \leftrightarrow B_{\lambda \mu}$ and $C \leftrightarrow C_{\lambda \mu}$ play the roles of the inertial mass parameter and stiffness-coefficient, respectively, here considered constant. Equilibrium deformations $a_{\lambda \mu}^{e q .}$ and/or $b_{\lambda \mu}^{e q .}$ are selected using our extensive Woods-Saxon Strutinsky results. Introducing the vibration frequencies $\hbar \omega \equiv \hbar \sqrt{C / B}$ and simplifying the notation we define $\eta \equiv \hbar /[\sqrt{2} B \omega]^{1 / 2}$ and introduce the usual harmonic-vibration solutions

$$
\phi_{n}(\rho)=\sqrt{\eta /\left(2^{n} n ! \sqrt{\pi}\right)} \exp \left(-\eta^{2} \rho^{2} / 2\right) H_{n}(\rho),
$$

where $H_{n}$ are Hermite polynomials. We wish to avoid mathematical details at this point and limit ourselves to schematizing the main lines of the approach; the interested reader is referred to a more detailed discussion in [9].

We use the standard definition of the intrinsic reference frame (alternatively: Euler angles) based on the choice of the quadrupole deformations as $\alpha_{2,+1}=0=\alpha_{2,-1}$ and $\alpha_{2,+2}=\alpha_{2,-2}$. In such a case the quadrupole vibrations are described in terms

$\S$ The negative parity bands with the above properties have been found in the literature, cf. a short review of the actual research status of the International TetraNuc (Tetrahedral Nuclei) Collaboration in [10]; they can be seen as tetrahedral-symmetry candidate structures.

|| To better appreciate this argument consider a nucleus with the quadrupole-deformed minimum of the order of $\alpha_{20} \approx 0.3$ and the induced collective rotation. The corresponding reduced transition probabilities, B(E2), could be as large as $300 \mathrm{~W} . \mathrm{u}$. depending of the nuclear mass while the correction of the zero-point vibrations of the order of a dozen W.u. or so. The same 'correction' applied in the case of the static, nearly-vanishing deformation [and therefore considerably smaller, nearly-vanishing $\mathrm{B}(\mathrm{E} 2)]$ may take the role of the leading term rather then that of a negligible correction. 
of two independent real parameters $\alpha_{20}=a_{20}$ and $\alpha_{22}=a_{22}$ and it turns out that the octupole vibrations represent seven independent modes, here parametrised with the help of $a_{30}$ as well as $a_{31}, a_{32}, a_{33}$ and $b_{31}, b_{32}, b_{33}$. In the following we would like to construct the negative-parity one-phonon wave-functions to be able to model the negative-parity bands, candidates for the tetrahedral symmetry, in terms of the irreducible representations of the underlying tetrahedral group. With $n$ in (12) representing the phonon number, we will introduce the symbol $\{n\} \equiv$ $\left\{n_{1}, n_{2} ; n_{3}, \ldots n_{9}\right\}$ in which the first two places are reserved for the quadrupole degrees of freedom and all the others of the octupole ones. We will introduce a basis of product wave-functions

$$
\Phi_{\{n\}}=\phi_{n_{1}} \phi_{n_{2}} \times \phi_{n_{3}} \ldots \phi_{n_{9}},
$$

but since the conditions for the negative parity solutions imply the one-phonon structure it follows that $n_{1}=0$ and $n_{2}=0$ (zero-phonon quadrupole ground-state) whereas $\sum_{i=3}^{i=9} n_{i}=1$, with $n_{i}=0$ or 1 . It will be possible (and convenient) to shorten the notation by replacing $\left\{n_{1}, n_{2} ; n_{3}, \ldots n_{9}\right\}$ by $\{00 ; m\}$, where $m$ gives the only nonzero index $n_{i}$ that is allowed. For instance, symbol $\Phi_{00 ; n_{5}}$ represents the product $\left(\phi_{n_{1}=0} \cdot \phi_{n_{2}=0}\right) \times\left(\phi_{n_{3}=0} \cdot \ldots \cdot \phi_{n_{5}=1} \cdot \ldots \cdot \phi_{n_{9}=0}\right)$.

Consider an arbitrary solution $\Psi$ to the vibration-part of Hamiltonian in (10); it can be expanded in terms of our basis wave-functions as

$$
\Phi=\sum_{j=3}^{9} c_{\{n\}_{j}} \Phi_{\{n\}_{j}} .
$$

Let $P^{\Gamma}$ denote the operator projecting from the space of $\Psi$ onto a sub-space of the irreducible representations $\Gamma$ of $G$; we will apply it for $G=T_{d}$, but the considerations remain analogous for any symmetry point-group. According to the well known result

$$
P^{\Gamma}=\frac{\operatorname{dim}[\Gamma]}{\operatorname{card}(G)} \sum_{\hat{g} \in G} \chi^{\Gamma}(\hat{g})^{\star} \hat{g},
$$

where $\operatorname{card}(G)$ is the number of elements in the group $G$ and $\chi^{\Gamma}(\hat{g})$ are the related characters of the group elements. Using the above operator we find the structure of the wave-functions spanning the irreducible representations of the group $\mathrm{T}_{d}$ in the 7-dimensional space of one-phonon solutions; up to a normalisation they are given by:

$$
|A 1\rangle \sim \Psi_{00 ; n_{6}}
$$

for the scalar representation $\Gamma=A 1$,

$$
\begin{aligned}
& |T 1 ; 1\rangle \sim \Psi_{00 ; n_{3}} \\
& |T 1 ; 2\rangle \sim \frac{1}{4}\left(-\sqrt{5} \Psi_{00 ; n_{2}}-\sqrt{3} \Psi_{00 ; n_{4}}-i \sqrt{5} \Psi_{00 ; n_{5}}+i \sqrt{3} \Psi_{00 ; n_{7}}\right) \\
& |T 1 ; 3\rangle \sim \frac{1}{4}\left(+\sqrt{5} \Psi_{00 ; n_{2}}+\sqrt{3} \Psi_{00 ; n_{4}}-i \sqrt{5} \Psi_{00 ; n_{5}}+i \sqrt{3} \Psi_{00 ; n_{7}}\right)
\end{aligned}
$$

for the first 3-dimensional representation $\Gamma=T 1$ and

$$
\begin{aligned}
& |T 2 ; 1\rangle \sim \Psi_{00 ; n_{1}} \\
& |T 2 ; 2\rangle \sim \frac{1}{4}\left(-\sqrt{3} \Psi_{00 ; n_{2}}+\sqrt{5} \Psi_{00 ; n_{4}}+i \sqrt{3} \Psi_{00 ; n_{5}}+i \sqrt{5} \Psi_{00 ; n_{7}}\right) \\
& |T 2 ; 3\rangle \sim \frac{1}{4}\left(+\sqrt{3} \Psi_{00 ; n_{2}}-\sqrt{5} \Psi_{00 ; n_{4}}+i \sqrt{3} \Psi_{00 ; n_{5}}+i \sqrt{5} \Psi_{00 ; n_{7}}\right)
\end{aligned}
$$

for the remaining one, $\Gamma=T 2$, all that for the vibrations around the spherical equilibrium in the 7 -dimensional octupole space. To obtain the solutions for the 
non-zero equilibrium deformation we may introduce the deformation-shift operators, say $\mathcal{S}$, that must preserve the group structure of $G$ - and thus must commute with all the symmetry operations in $G$. It is possible to show that the only invariant of the $G=T_{d}$ group composed of the $\alpha_{\lambda=3, \mu}$ tensor can be expressed (depending on conventions that are of no importance here) as either $b_{32}$ or $a_{32}$. Using the first convention we may demonstrate that the 'tetrahedral-deformation shift' operator with the sought mathematical properties has the form

$$
\mathcal{S}\left(b_{32}^{e q \cdot}\right)=\exp \left(-b_{32}^{e q \cdot} \frac{\partial}{\partial b_{32}}\right)
$$

Introducing the above shift we formally break the symmetry under the inversion $\uparrow$. In such a case the projection onto the good parity is an option of choice. It is easy to see that the operator $\frac{1}{2}\left(1-\mathcal{C}_{i}\right)$, where $\mathcal{C}_{i}$ denotes the inversion, is the sought projection on the negative-parity irreducible-representations of the considered $T_{d}$-group and thus by applying the combined projection-and-shift operation

$$
\mathcal{P}\left(b_{32}^{e q \cdot}\right) \equiv \frac{1}{2}\left(1-\mathcal{C}_{i}\right) \mathcal{S}\left(b_{32}^{e q \cdot}\right)
$$

on the wave functions in (16-22) we obtain the sought solutions that belong to the irreducible representations of the group considered. They have the appropriately adjusted equilibrium deformations and are also parity-projected.

We have discussed so far, for the sake of compactness of this presentation, only the main lines of the proposed approach, but the interested reader can convince her/himself that due to the simplicity of the vibrational wave-function basis the calculations of interest can be performed analytically ${ }^{+}$; this is a helpful factor for quick estimates but by no means the most important one. As a first example of an application (and using the fact that the exotic deformations considered here are relatively small) we may introduce the general expansion expression for the multipole moment operators

$Q_{\lambda \nu}^{r o t}=\frac{3 Z R_{0}^{\lambda}}{4 \pi}\left\{\alpha_{\lambda \nu}+\frac{\lambda+2}{2 \sqrt{4 \pi}} \sum_{\lambda_{1} \lambda_{2}} \sqrt{\frac{\left(2 \lambda_{1}+1\right)\left(2 \lambda_{2}+1\right)}{2 \lambda+1}} \times\left(\lambda_{1} 0 \lambda_{2} 0 \mid \lambda 0\right)\left(\alpha_{\lambda_{1}} \otimes \alpha_{\lambda_{2}}\right)_{\lambda \nu}\right\}$,

valid for the simplified uniform charge distributions in the nuclear intrinsic ('rotating') reference frame. The laboratory-frame image of this operator is

$$
Q_{\lambda \mu}^{l a b}=\sum_{\nu} D_{\mu \nu}^{\lambda}(\Omega)^{\star} Q_{\lambda \nu}^{r o t}
$$

where $D_{\mu \nu}^{\lambda}(\Omega)^{\star}$ are the complex-conjugate Wigner functions. Using theorems of the group-representation theory and the fact that the assumed Hamiltonian does not couple the rotations and the vibrations we may construct the final solutions in the group-theory adapted form

$$
\Psi_{\Gamma j \sigma ; J M \nu}^{(\lambda=2,3)}(\alpha, \Omega) \sim \Phi_{\Gamma j \sigma}^{(\lambda=2,3)}(\alpha) R_{J M \nu}(\Omega),
$$

9 We encounter here a certain mathematical artifice related to the fact that we have chosen to illustrate the parity-projection technique. The use of the latter in this particular case is not really necessary, since the potential energies are in fact always even functions of $\alpha_{\lambda=3, \mu}$ variables. However, the projection technique presented here has certain merits for its applicability in more general situations and we discuss it for that reason.

+ An introduction of the realistic potentials generated using the mean-field theory leads to the condition $V\left(-\alpha_{\lambda=3, \mu}\right)=V\left(+\alpha_{\lambda=3, \mu}\right)$, (even functions) and the corresponding solutions are not proportional just to a single Hermite-polynomial but rather to a series of such functions and must be obtained numerically. 
where $\alpha \equiv\left\{\alpha_{2} \otimes \alpha_{3}\right\}$. Above, $R_{J M \nu}(\Omega)$ represents the rotational part of the wavefunction while quantum numbers $J M$ represent the total angular momentum of the system together with its projection and $\nu$ distinguishes among the $(2 \mathrm{~J}+1)$-solutions of the rotor Hamiltonian that correspond to a given $(J M)$. The phonons can also be characterised by the associated quantum numbers $\mathrm{jm}$, analogous with respect to $J M$. In the solutions that were presented in (16-22), (and functions $\Phi_{\Gamma j \sigma}^{(\lambda=2,3)}(\alpha)$ are constructed out of them) the involved three-dimensional irreducible representations contain in general the combinations of the $m$-components. It then follows that the resulting wave functions need to be labelled by a separate index, $\sigma$, enumerating states within the three-dimensional irreducible representations. Finally, the symbol $\Gamma$ refers to irreducible representations of the group $T_{d}$, above denoted $A 1, T 1$ and $T 2$, that possibly belong to different irreducible representations of the $\mathrm{SO}(3)$-group, the latter enumerated with $j$.

We are going to limit ourselves here to presenting some instructive estimates of the expectation values of selected charge moments using the irreducible-representation form of the vibrational solutions. Calculations using the elements of the approach presented so far and assuming for simplicity that all the $\eta_{3 \mu}=\eta_{3}$ give

$\left\langle A 1\left|Q_{20}\right| A 1\right\rangle=\left\langle T 1 ; 1\left|Q_{20}\right| T 1 ; 1\right\rangle=-\left\langle T 2 ; 1\left|Q_{20}\right| T 2 ; 1\right\rangle=-\frac{Z R_{0}^{2}}{2 \pi \sqrt{5 \pi}} \cdot \frac{1}{\eta_{3}^{2}}$.

This is instructive (although at this stage quite schematic) a result stressing once again the importance of the zero-point motion mechanism in the symmetry considerations. In words: the above results suggest that the tetrahedral-symmetry solutions contribute to the quadrupole transitions through a dynamical (oscillation) mechanism involving all the octupole components. This is in contrast to the earlier suggestions based on the pure static considerations and implying that the quadrupole transitions in the exact symmetry limit should vanish exactly.

Let us observe that the above conclusion is certainly only one on a long list. The issues that remain Open Problems to us are: a. Complete (and not only schematic) calculations of the reduced transition probabilities $B(E 2 ; \Gamma \rightarrow \Gamma), B\left(E 2 ; \Gamma \rightarrow \Gamma^{\prime}\right)$ and: b. The ratios $B(E 2 ; \Gamma \rightarrow \Gamma) / B(E 1 ; \Gamma \rightarrow g s b)$ and $B(E 2 ; \Gamma \rightarrow \Gamma) / B\left(E 2 ; \Gamma \rightarrow \Gamma^{\prime}\right)$ together with the related combinations of stretched and non-stretched E2 and E1 transitions. Symmetries act very restrictively on the rules for inter-band transitions depending on the type of an irreducible representation and the transition kind, giving characteristic fully forbidden or retarded transitions, alternatively characteristic branching ratios - that can be used to distinguish one symmetry from another.

\section{Level-mixing models as a totally unsuited tool to search for symmetries}

There exists an exercise in quantum mechanics dubbed two-level mixing model, cf. [11] for probably the most rigorous presentation, and also [12]. The model has been introduced to nuclear structure physics in the context of rotational-band mixing and the Coriolis mixing, cf. [13, 14, 15], and references therein. In nuclear structure it has been successful in parametrising certain features in the presence of strong quadrupole deformations and dominating E2 transitions, but in the mean time used gradually less and less properly, its limiting assumptions ignored or presumed valid without verification, no attention payed to parametric instabilities etc.

In testing the symmetries, the signals are expected to be weak and so are the related electromagnetic transition matrix elements expected to be of the order of 
those which are usually neglected in 'level-mixing' articles known to us. Moreover, all exotic symmetries treated in this text involve non-axial shapes and thus using the assumption of axiality (good $K$ quantum number, the 'usual' assumption) is totally incorrect. Anticipating that in the present context the level-mixing model may again be a tempting option to replace partly or entirely the thorough measurements we briefly add a few more among the most relevant inadequacies of this 'method' in studying symmetries.

Let us emphasize that no calculation in general and the level-mixing calculations in particular should ever be used in place of a measurement. However, in our opinion, much too often the information deduced from the level-mixing calculations forces its way down to the conclusions as if it were the experimental result.

In which respect the quantum-mechanics exercise of two-level mixing poses an Open Problem in nuclear physics of the $\mathrm{XXI}^{\text {st }}$ Century? We will reformulate this question: "How can we lower the damage to the experimental research caused by the model's in-appropriate use?" In commenting these issues we let ourselves being guided by the observations made during recent discussions, conference presentations and even through recent publications!

In order to establish the presence of a symmetry it is important to verify the symmetry criteria e.g. the ones based on the electromagnetic transition probabilities or, if that is not fully possible, certain specific branching ratios as discussed in section 3. This task involves inevitably weak transitions that may be difficult to measure. The band mixing models have been used in the past to replace the weak experimental signal from, say band $B$, by the results obtained through a mixing model calculations provided some information about the electromagnetic transitions has been experimentally available for another band, say $A$, the so-called reference band.

In their excellent presentation entitled "The theory of nuclear level mixing resonant spectroscopy", [16], the authors begin by justly emphasizing the fact that the level-mixing models are based by construction on the symmetry breaking mechanism. Indeed, according to Landau-Zener rule, two bands cross because their states differ in symmetry - they repel each other because there is an interaction that causes the state mixing. More precisely: given states $\phi_{a}$ and $\phi_{b}$ belonging either to two different irreducible representations of the symmetry group of one generating Hamiltonian $h_{g}$ or obtained from two distinct generating Hamiltonians $h_{g}^{\prime}$ and $h_{g}^{\prime \prime}$. In order that the states mix it is necessary that the mixing interaction $v_{m}$ does not commute, neither with $h_{g}$ nor with at least one of the other two Hamiltonians. In other words, the ' full' Hamiltonian $h=h_{g}+v_{m}$ or $h=h_{g}^{\prime}+h_{g}^{\prime \prime}+v_{m}$ generates solutions of lower symmetry since $\left[h, v_{m}\right] \neq 0$, and none of the interacting states has its initial symmetry.

Since the level-mixing Hamiltonian breaks the original symmetries of each of the mixed states, it becomes clear that the band mixing model is from the very beginning not suited to look for symmetries. It then follows that e.g. complicating the same model by adding more and more mixing bands would be multiplying inadequacies and possibly even increasing the existing confusion.

\section{Point-groups symmetries and nuclear stability: Open Problems}

In this article we have formulated in fact two strategies in searching for the so-called geometrical (synonymous with the point-group) symmetries in Nuclear Structure. They are based on the group-theory guided discussion of the problems: What are the nuclear geometries that privilege increased stability of the nuclear N-body systems? 
and: Where in the Periodic Table such symmetries can possibly be manifested? The second strategy addresses the issue of the experimental criteria: What should be measured in order to establish the presence (or absence) of the point-group symmetries?

In discussing the first series of the implied Open Problems we have introduced the notion of the generalized (as compared to the standard, spherical one) shellstructures that can be associated with any symmetry group of the Hamiltonian not necessarily the 'usual' spherical symmetry. The first observables of interest in this case are the 'magic gap chains' - the series of large energy gaps in the singlenucleon spectra that express the system-stabilizing response of the nuclear mean field to the privileged geometrical shapes: those manifesting the point-group symmetries. On the basis of the earlier publications we have formulated a qualitative criterion suggesting that the symmetries expressed by the point groups which have numerous irreducible representations and/or whose irreducible representations have possibly largest dimensions are likely to produce the strongest non-spherical shell effect. The systematic although in a way still preliminary studies were focussed so far on the tetrahedral and octahedral symmetries, with theoretical effort accompanied by the series of dedicated experiments performed within the TetraNuc collaboration. But examining the whole series of nearly a dozen of point groups of special interest listed in this article is an Open Problem for this domain of research.

The second series of Open Problems is related to the research of observables and the construction of the adequate experimental methods. We have proposed an adaptation of the well known, and again a group-theory based approach, that consists in calculating the electromagnetic transition probabilities in function of the pointgroup symmetry. According to this suggestion the solutions of the collective rotationvibration Hamiltonian whose eigenfunctions are expressed in terms of the irreducible representations of the involved symmetry groups are used to predict the properties of the electromagnetic transitions and related branching ratios. Such transitions vary, often dramatically, depending on the symmetry group involved and can be employed as valuable criteria - however, they were not used so far in the search for symmetries.

We have also re-visited the level-mixing models that can occasionally be used to fit and/or estimate the experimental data otherwise difficult to really measure. Since these models have also been used in the past as substitutes for the experimental research we have re-examined the model's functioning and formulated rather obvious, strong warning: the model's unknowns and the applicability conditions may be more difficult to establish experimentally than the attempted measurement itself.

\section{Acknowledgements}

The research programme and associated Open Problems reported here are related to the activities of the TetraNuc (Tetrahedral Nuclei) Collaboration involving 18 institutions worldwide. This informal collaboration supported in part by IN2P3, France, aims at searching for the fingerprints of the tetrahedral symmetry throughout the Nuclear Chart. The authors want to warmly thank all who have taken part in these activities and more specifically the Direction of the IPN-Orsay and D Verney, D Curien, the co-spokesman of the collaboration as well as O Stézowski and Q T Doan at IPN-Lyon, J Gerl at GSI-Darmstadt, R Julin and P Jones at JYFL-Jyvaskyla, F Haas, P Medina and M Richer at IPHC-Strasbourg, M Jentschel, B Lauss and W Urban at ILL-Grenoble, D Tonev at INRNE-Sofia, G de Angelis at LNL-Legnaro, 
R J Singh at IUAC-New Dehli, L Riedinger at University of Tennessee at Knoxville, D Hartley at U.S. Naval Academy-Annapolis, and last but not least the Direction of the ANL-Argonne, USA and the Direction of the IN2P3, France.

\section{References}

[1] Molique H, Dudek J, Góźdź A and Mazurek K Xarchiv ..... and to be published

[2] Cornwell J F 1984 Group theory in physics (London, Academic Press)

[3] Dudek J, Dobaczewski J, Dubray N, Góźdź A, Pangon V and Schunck N 2007 Int. J. of Mod. Phys. E E16 516

[4] Dudek J, Góźdź A, Schunck N and Miśkiewicz M 2002 Phys. Rev. Lett. 88 252502; Góźdź A and Dudek J 2007 Int. J. Mod. Phys. E 16541

[5] Schunck N, Dudek J, Góźdź A and Regan P H 2004 Phys. Rev. C 69 061305(R)-1

[6] Dudek J, Curien D, Dubray N, Dobaczewski J, Pangon V, Olbratowski P and Schunck N 2006 Phys. Rev. Lett. 9707250

[7] Dudek J, Góźdź A and Schunck N 2003 Acta Phys. Polonica B 342491

[8] Dudek J, Mazurek K, Curien D, Dobrowolski A, Góźdź A, Hartley D, Maj A, Riedinger L and Schunck N 2009 Acta Phys. Polonica B 40713

[9] Góźdź A, Dobrowolski A, Dudek J and Mazurek K 2010 Int. J. Mod. Phys. E, in print

[10] Curien D, Dudek J and Mazurek K 2010 J. Phys. G (Conference series) in print

[11] Cohen-Tannoudji C, Diu B and Laloë F 1986 Mécanique Quantique vol 1, p. 418 (Paris: Hermann)

[12] Davydov A S 1965 Quantum Mechanics (Oxford: Pergamon)

[13] Rowe D J 1970 Nuclear Collective Motion (London: Methuen)

[14] Bohr A and Mottelson B R 1975 Nuclear Structure vol 2 (Reading: Benjamin)

[15] Casten R F 1990 Nuclear Structure from a Simple Perspective (Oxford, Oxford University Press)

[16] Coussement R, Put P, Scheveneels G, and Hardeman F 1985 Hyp. Int. 23309 